\title{
Multivessel CORONARY ARTERY SPASM IN PERICARDITIS
}

Singapore Med J 2018; 59(11): 611-613 https://doi.org/10.11622/smedj.2018138

Dear Sir,

Coronary vasospasm can result in tombstone-like ST elevations in the presence of non-obstructive coronary arteries on angiography. Coronary vasospasm has been reported to coexist with myocarditis, possibly due to endothelial dysfunction or coronary smooth muscle cell hyperreactivity. We herein describe a novel presentation of tombstone-like ST elevations that were likely from multivessel coronary vasospasm in a patient with pericarditis.

A 40-year-old man with a three-day history of an infected right lower molar tooth presented to the emergency department with dysphagia, shortness of breath and right neck swelling. There was no complaint of chest pain, and he had no past medical history or any cardiac problems prior to presentation. Electrocardiography (ECG) performed at the point of admission showed typical global concave upward ST elevations with PR interval depression consistent with pericarditis (Fig. 1a). Further investigations revealed elevated inflammatory markers. Computed tomography of the neck and thorax, which was performed in view of his presentation history, showed gas gangrene in the neck communicating with the mediastinum, likely due to contiguous infection.

The patient underwent surgery for his disease and received broad-spectrum antibiotics, as guided by microbiological cultures. On the sixth day of admission, diffuse tombstone-like non-territorial ST elevations were seen on ECG without any symptom or haemodynamic instability (Fig. 1b); they completely resolved after 15 minutes. There were no Q waves thereafter. The diffuse tombstone-like ST elevations recurred on the tenth day of admission and again resolved after 15 minutes. Peak serum troponin levels were marginally elevated at $0.063 \mathrm{mcg} / \mathrm{L}$ (normal $<0.040 \mathrm{mcg} / \mathrm{L}$ ). The patient was under sedation and intubated for the first two weeks of his admission.
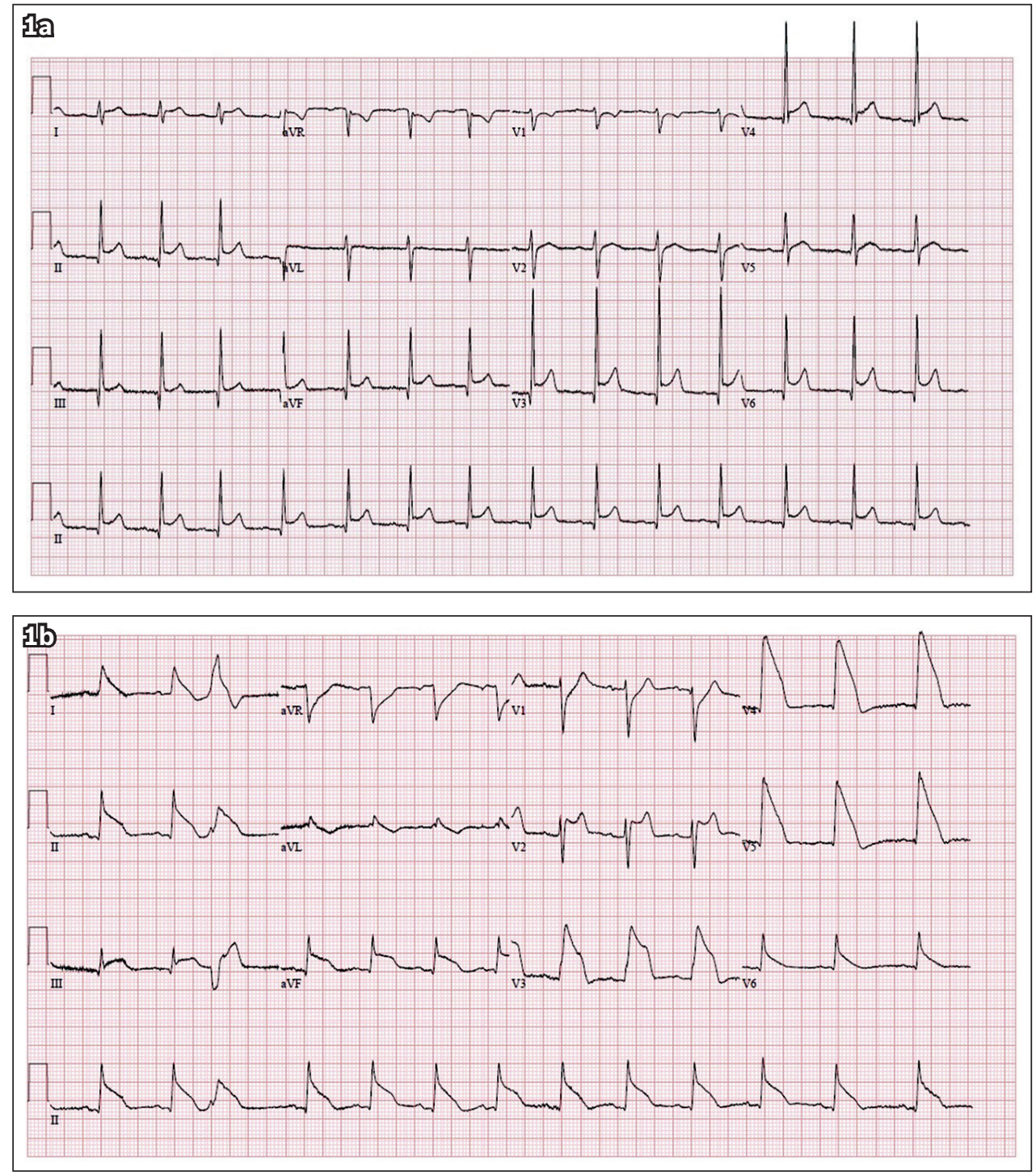

Fig. 1 ECGs shows (a) global concave upward ST elevations with PR interval depressions secondary to pericarditis; and (b) transient tombstone-like non-territorial ST elevations consistent with transmural injury. 
Urgent coronary angiography showed normal coronary arteries. Subsequent transthoracic echocardiography showed normal left ventricular ejection fraction with no regional wall motion abnormalities. There was no obvious pericardial thickening or pericardial effusion of note. Cardiac magnetic resonance (CMR) imaging showed a thickened pericardium and a small pericardial effusion consistent with pericarditis (Fig. 2). There was no high T2-weighted signal on short tau inversion recovery images or late gadolinium enhancement images that could suggest myocardial oedema or inflammation (related to myocarditis or myocardial infarction). A review of the patient's medication did not show any drugs known to incite vasospasm and his electrolytes were unremarkable throughout the admission. He had no history of any prior medication use, including illicit drugs such as cocaine.

The patient was managed with anti-inflammatory medication and coronary vasodilators, and continued treatment for his underlying gas gangrene. He was asymptomatic throughout admission. There were no further episodes of ST elevations. The patient made a full recovery and was subsequently discharged well for outpatient follow-up. One month later, ECG showed global T wave inversions consistent with full evolution of pericarditis (Fig. 3).

This case demonstrates non-territorial tombstone-like ST elevations in a previously healthy man admitted with pericarditis from contiguous spread of infection from a right lower molar. His preceding ECG had the classic changes of pericarditis. These tombstonelike ST elevations were transient (only lasting for 15 minutes before complete resolution), widespread and not limited to any single coronary artery territory. This is not consistent with transmural infarctions, which usually affect one or at most two coronary vascular territories and frequently manifest with ST elevations that evolve over hours, resulting in Q waves. Coronary angiography showed normal coronaries with no evidence of obstructive coronary artery disease.

Stress-induced (Takotsubo) cardiomyopathy can cause similar transient changes, ${ }^{(1,2)}$ but this is ruled out by a normal transthoracic echocardiogram. Another consideration is resolved coronary thrombi. However, it would be unusual to have simultaneous thrombi formation and resolution in all coronary arteries. Although this patient was not on propofol, propofol infusion syndrome would be

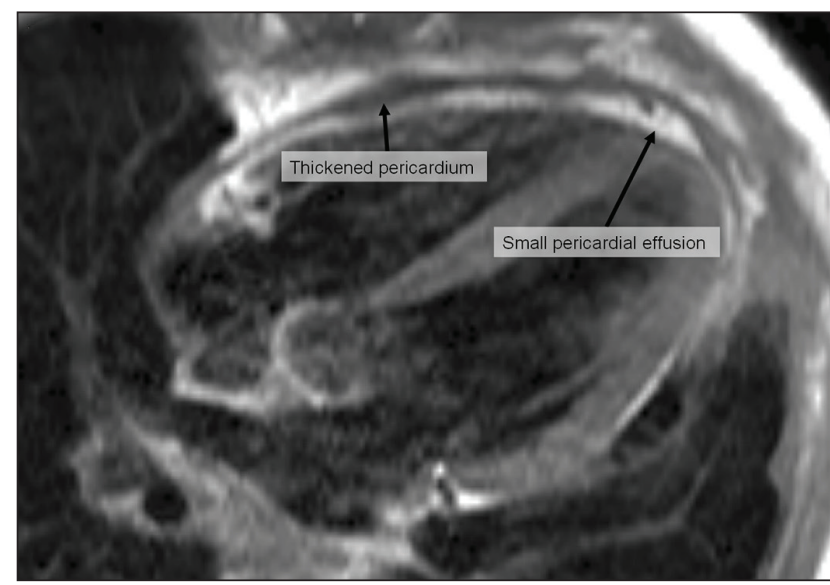

Fig. 2 Cardiac magnetic resonance image shows a thickened pericardium and a small pericardial effusion due to pericarditis. No myocardial oedema or inflammation was demonstrable on short tau inversion recovery images or late gadolinium enhancement images. a valid consideration for patients under anaesthesia and can cause similar ECG changes, which are typically described as Brugada-like. ${ }^{(3)}$ The ECG changes of this patient were not typical of other differentials of ST elevation, such as early repolarisation, left ventricular hypertrophy, hyperkalaemia and Brugada syndrome. ${ }^{(4)}$ The patient's CMR image confirmed the diagnosis of pericarditis without myocarditis. In this setting, the transient diffuse tombstone-like ECG changes are most consistent with multivessel coronary vasospasm due to pericarditis.

The association between myocarditis and coronary artery spasm is well described. ${ }^{(5-10)}$ In a study of 85 patients with a clinical diagnosis of myocarditis but no obstructive coronary artery disease, inducible coronary artery vasospasm was proven to be more prevalent in patients with biopsy-proven evidence of myocarditis (myocardial inflammation or viral genome) than in those without. A strong association was found between parvovirus B19 myocarditis and coronary vasospasm, with around $86 \%$ of

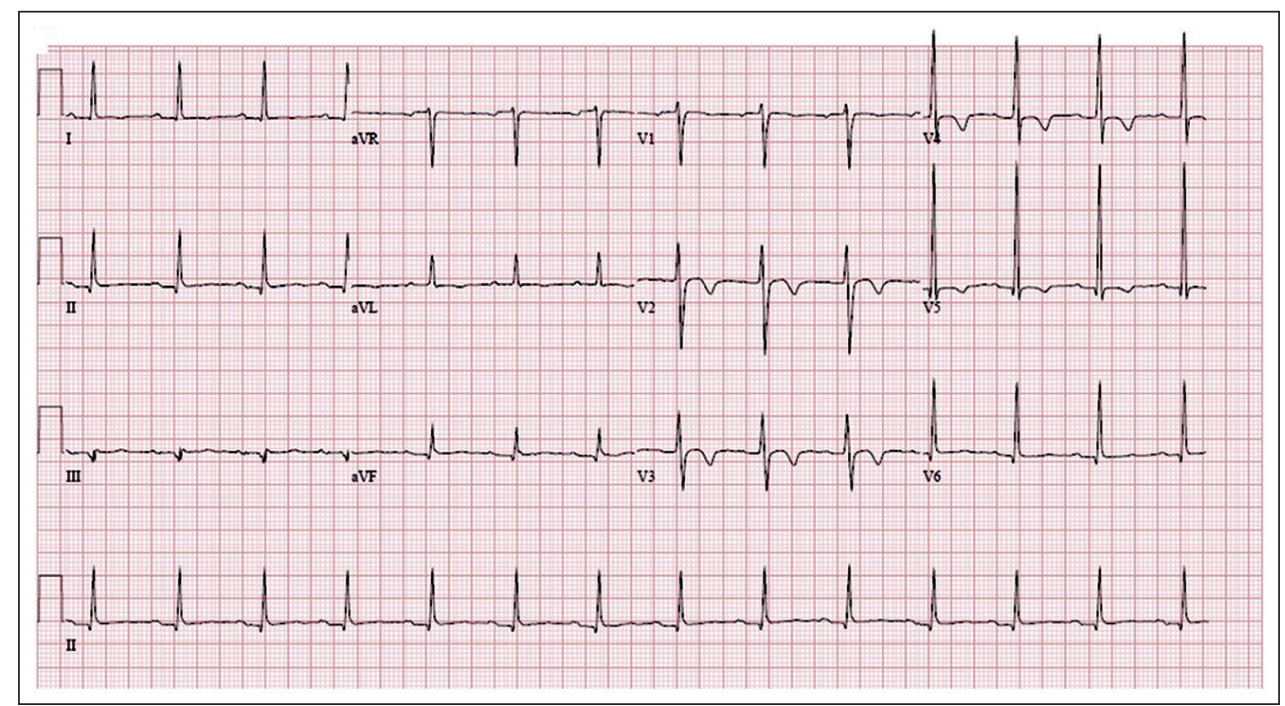

Fig. 3 ECG shows global T-wave inversions with resolution of ST elevations, consistent with evolution of pericarditis. 
patients with proven myocarditis demonstrating coronary vasospasm. The authors hypothesised that the underlying pathophysiology could be inflammation leading to endothelial dysfunction or coronary smooth muscle cell hyperreactivity. ${ }^{(11)}$ Although vasospasm has not been described in patients with pericarditis, myocarditis and pericarditis are inherently related and it is likely that pericarditis can incite similar changes.

The ECG changes seen in our patient are similar to those of patients with proven multivessel coronary vasospasm. ${ }^{(12,13)}$ In a study of patients with suspected vasospastic angina, diffuse multivessel involvement was described to be present in the majority of patients who demonstrated coronary vasospasm. ${ }^{(14)}$ The involvement of the entire pericardial sac, as evident in this patient, increases the likelihood of multivessel coronary inflammation and, by extension, multivessel vasospasm. Multivessel coronary artery spasm has

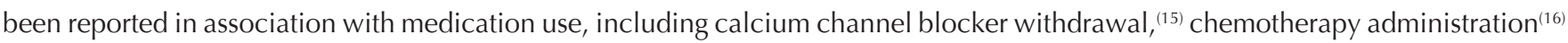
and possibly nitrate withdrawal. Sequelae of coronary artery spasm can include cardiogenic shock and arrhythmic complications. ${ }^{(13,17)}$ Management includes coronary vasodilators and supportive management for any cardiac complications. To our knowledge, this is the first case report that describes multivessel coronary vasospasm in a patient with pericarditis.

Yours sincerely,

Yinghao $\underline{\operatorname{Lim}}^{1}$, Devinder $\underline{\operatorname{Singh}}{ }^{1}$, Poay Huan $\underline{\mathrm{Loh}}^{1}$, Kian Keong $\underline{\mathrm{Poh}}^{1}$

${ }^{1}$ Department of Cardiology, National University Heart Centre, Singapore. yinghao_lim@nuhs.edu.sg

\section{References}

1. Angelini P. Transient left ventricular apical ballooning: a unifying pathophysiologic theory at the edge of Prinzmetal angina. Catheter Cardiovasc Interv 2008; $71: 342-52$.

2. Sia CH, Singh D, Loh JP. Can you interpret the ECG under stress? Singapore Med J 2018; 59:455-9.

3. Riera AR, Uchida AH, Schapachnik E, et al. Propofol infusion syndrome and Brugada syndrome electrocardiographic phenocopy. Cardiol f 2010; 17:130-5.

4. Wang K, Asinger RW, Marriott HJ. ST-segment elevation in conditions other than acute myocardial infarction. N Engl J Med 2003; 349:2128-35.

5. Iwasaki K, Kusachi S, Tominaga Y, Kita T, Taniguchi G. Coronary artery spasm demonstrated by coronary angiography in a patient with acute myocarditis resembling acute myocardial infarction; a case report. Jpn I Med 1991; 30:573-7.

6. McCully RB, Cooper LT, Schreiter S. Coronary artery spasm in lymphocytic myocarditis: a rare cause of acute myocardial infarction. Heart $2005 ; 91: 202$.

7. Ferguson DW, Farwell AP, Bradley WA, Rollings RC. Coronary artery vasospasm complicating acute myocarditis. A rare association. West J Med 1988; $148: 664-9$.

8. Rowe MK, Rutherford M, Kostner K. An unusual cause of ST elevation: coronary vasospasm complicating acute myocarditis - a case report and review of the literature. J Clin Prev Cardiol 2016; 5:146-8.

9. Gibelli G, Devizzi S, Brioschi P, Rimini A, Biasi S. Sudden ST elevation with angina-like pain in myocarditis. An uncommon course of a common disease: strategic role of cardiac magnetic resonance. J Cardiovasc Med (Hagerstown) 2009; 10:264-6.

10. Silva D, Marques P, Martins S, et al. Coronary artery vasospasm and acute myocarditis: a rare association. Rev Port Cardiol 2010; $29: 1879-88$.

11. Yilmaz A, Mahrholdt H, Athanasiadis A, et al. Coronary vasospasm as the underlying cause for chest pain in patients with PVB19 myocarditis. Heart 2008; $94: 1456$ 63.

12. Richardson JD, Nelson AJ, Worthley SG, et al. Multivessel coronary artery spasm. Heart Lung Circ 2012; 21:113-6.

13. Ahn J, Kim B, Park H, Park K, Kim YD. Cardiogenic shock from global myocardial ischemia induced by simultaneous multivessel coronary spasm. Korean Circ J 2012; 42:427-30.

14. Park YM, Han SH, Ko KP, et al. Diffuse multi-vessel coronary artery spasm: incidence and clinical prognosis. Int J Cardiol 2013; 167:398-402.

15. Kostis WJ, Suh WM, Palacios IF. Acute myocardial infarction caused by multivessel coronary spasm due to calcium channel blocker withdrawal. Catheter Cardiovasc Interv 2011; 78:229-33.

16. Naib T, Steingart RM, Chen CL. Sorafenib-associated multivessel coronary artery vasospasm. Herz 2011; 36:348-51.

17. Bromberg-Marin G, Mahmud E, Tsimikas S. Spontaneous multivessel coronary vasospasm leading to cardiogenic shock. J Invasive Cardiol 2007; 19 :E85-8. 